\title{
Estado Nutricional de Escolares da Rede Pública de Ensino de Piedade, SP*
}

\author{
Giovana Eliza Pegolo ${ }^{1}$, Marina Vieira da Silva ${ }^{2}$
}

\begin{abstract}
A pesquisa visou avaliar o estado nutricional de escolares da rede pública de ensino do município de Piedade, Estado de São Paulo. A amostra foi constituída por 150 escolares, de ambos os gêneros, com idade entre 7 e 14 anos. Para a análise da situação nutricional, foram utilizadas as medidas antropométricas de peso e altura. Os resultados mostraram que a proporção de $4 \%$ de escolares foi classificada com indicativo de déficit de altura, percentual considerado superior ao esperado $(2,3 \%)$. No intervalo $(-2 \leq Z \mathrm{AI}<-1)$ que discrimina os escolares com indicativo de comprometimento da altura, foram identificados $19,3 \%$, enquanto a proporção esperada é 13,6\%. Quanto às análises do IMC, foram observados 10,7\% de escolares com baixo peso, enquanto na situação oposta (obesidade) a proporção verificada alcançou 1,6\% dos integrantes da amostra. Os resultados obtidos diferem daqueles observados quando são examinados os dados entre estudantes, também matriculados na rede pública de ensino, de substancial número de municípios paulistas. Nesta pesquisa, a proporção de escolares classificados com baixo peso supera a prevalência de escolares com obesidade. Análises que envolvam, além da população urbana, também aquela moradora do meio rural, tornam-se valiosas por constituírem subsídios para o planejamento de ações efetivas de saúde pública.
\end{abstract}

Palavras-Chave: antropometria, escolares, estado nutricional, obesidade

\section{Nutritional Status of Students of the Public School of the City of Piedade, SP}

This research aimed at evaluating the nutritional status of students of the public schools of Piedade, Sao Paulo State. The sample was constituted of 150 students, of both genders, with ages between 7 and 14 years. Anthropometrical measurements of weight and height were used to assess the nutritional situation. The results showed that the proportion of $4 \%$ of students was classified as having a height deficit, a proportion considered higher than expected (2.3\%). In the interval $-2 \leq$ $H A Z<-1$, that discriminates the population under risk of height-for-age deficit, $19.3 \%$ of the students were identified, against the expected proportion of $13.6 \%$. With regard to the analyses of BMI, $10.7 \%$ of students were found to be underweight, while the proportion of obese students was only 1.6\%. The results obtained differ from those of students enrolled in a substantial number of public schools in other cities of the state of Sao Paulo. In this study, it was evident that the proportion of students with low weight exceeds the prevalence of students with obesity. Analyses that involve not only the urban but also the rural population should provide solid data upon which to plan more effective public health actions.

\footnotetext{
${ }^{*} \mathrm{O}$ trabalho integra a pesquisa "Contrastes regionais nos custos, qualidade e operacionalização do Programa Nacional de Alimentação Escolar - PNAE e seu impacto sobre os padrões alimentares da população brasileira”, financiada pelo Conselho Nacional de Desenvolvimento Científico - CNPq (Processo no 504369/2003-2) e baseia-se na dissertação "Condicionantes do estado nutricional de escolares da rede pública de ensino em município com agricultura familiar", elaborada pela primeira autora.

${ }^{1}$ Faculdade de Ciências Farmacêuticas (UNESP).

${ }^{2}$ Escola Superior de Agricultura "Luiz de Queiroz" (USP).
} 
Key Words: Anthropometry, nutritional status, students, obesity

\section{Introdução}

Transformações econômicas, sociais e demográficas atingiram a sociedade brasileira nas últimas décadas e modificaram o perfil nutricional e educacional da população. A desnutrição, principalmente quando acomete os indivíduos mais jovens, continua despertando a preocupação de autoridades sanitárias e pesquisadores da área da saúde infantil ${ }^{[1]}$. Neste sentido, de acordo com Monteiro ${ }^{[2]}$, as crianças são biologicamente mais vulneráveis às deficiências nutricionais e, desta forma, são escolhidas como grupo indicador da presença de desnutrição na população. Conforme o referido autor, o percentual de crianças com retardo de crescimento (primeira e mais precoce manifestação de desnutrição na infância) reflete o risco de deficiências nutricionais a que está exposta uma coletividade.

Um aspecto considerado essencial para a compreensão do cenário epidemiológico dos problemas alimentares/nutricionais encontra-se nas disparidades regionais de renda. Notam-se contrastes marcantes na distribuição de rendimentos entre a população do meio rural, relativamente mais pobre, e a do meio urbano, especialmente quando consideradas as diferenças das regiões Norte e Nordeste do país ${ }^{[3]}$. Além disso, é possível observar, a partir de estudos que diferenciam a população rural da população urbana pobre, que as situações envolvendo a saúde, a nutrição e as condições de pobreza são piores no campo, constituindo um dos fatores para o êxodo rural ${ }^{[4]}$.

Contudo, embora diante de condições adversas, nas últimas três décadas, foi observada no Brasil expressiva redução nos valores de prevalência de deficiência estatural. Entre as crianças do meio urbano, a diminuição alcançou $72 \%$ e entre àquelas residentes em áreas rurais, de acordo com Batista Filho \& Rissin ${ }^{[3]}$, a redução atingiu 54,4\%. No espaço rural, este declínio mostrou-se bem menos acentuado, revelando aumento na disparidade nos quadros de prevalência da desnutrição entre crianças do meio urbano em relação àquelas moradoras do meio rural ${ }^{[3,5]}$.

Entretanto, outra situação que começa a despertar preocupação nos especialistas refere-se ao excesso de peso. Uma das explicações para o fenômeno destaca os fatores nutricionais inadequados decorrentes da transição nutricional. A transição é caracterizada por um aumento excessivo do consumo de alimentos ricos em gorduras e com elevado valor energético, aliado ao sedentarismo, ou seja, por mudanças no estilo de vida, determinadas por fatores culturais, sociais e econômicos ${ }^{[6]}$. No Brasil, os dados da Pesquisa de Orçamentos Familiares 2002-2003 (POF), realizada pelo Instituto Brasileiro de Geografia e Estatística (IBGE, 2006), revelam que a proporção de crianças e adolescentes com excesso de peso alcança 16,7\% ${ }^{[7]}$.

Cabe lembrar que a obesidade também é uma forma de má nutrição, associada ao surgimento de diversas comorbidades ${ }^{[8]}$. Pesquisas revelam que cerca de $60 \%$ das crianças e adolescentes com sobrepeso apresentam pelo menos um fator de risco adicional para doenças cardiovasculares, como hipertensão e hiperlipidemia ${ }^{[8,9]}$.

No Brasil, o acervo de pesquisas e análises relativo à juventude ainda é escasso e refere-se, sobretudo, às experiências juvenis dos grandes centros urbanos. Na maioria das vezes, não é levada em consideração a diversidade que caracteriza a infância e nem a adolescência vivenciada pela população residente na zona rural ${ }^{[10]}$.

Entre os principais objetivos desta pesquisa, destaca-se a avaliação do estado nutricional, por meio de indicadores antropométricos, de crianças e adolescentes matriculados na rede pública de ensino, moradores do município de Piedade (SP).

\section{Metodologia}

A pesquisa foi realizada no município de Piedade, Estado de São Paulo, cuja área territorial é de $746 \mathrm{~km}^{2}$. O município mantém atividades predominantemente classificadas no setor agrícola. De acordo com o Censo Populacional de 2000 (IBGE), Piedade possuía 50.131 habitantes. Deste total, $28.074(56,0 \%)$ residiam na zona rural e 22.057 
$(44,0 \%)$ na zona urbana ${ }^{[11]}$

Conforme resultados do Censo Escolar de 2004, divulgados pelo Instituto Nacional de Estudos e Pesquisas Educacionais Anísio Teixeira - INEP (2005), Piedade contava com 8.035 alunos regularmente matriculados nas redes estadual e municipal de ensino, sendo 4.096 de 1a a 4르 séries e 3.939 de $5^{a}$ a $8^{a}$ séries, perfazendo um total de 25 unidades de ensino ${ }^{[2]}$.

Para a seleção da amostra, as escolas (ensino fundamental e médio) foram sorteadas de maneira que a probabilidade de uma escola ser incluída na amostra fosse proporcional ao seu número de alunos. Para isso, foi elaborada uma tabela com o total das escolas do município, efetuando-se a soma (acumulada) do número de alunos, de maneira que cada unidade de ensino ficasse associada a um intervalo de valores da freqüência acumulada de escolares.

Seja $N$ o número total de alunos de todas as escolas consideradas no município, foi gerado um número aleatório inteiro de 1 a $N$. Em seguida, verificou-se qual era a escola cujo intervalo de freqüência acumulada incluía esse número inteiro obtido. Esta escola passou a integrar a amostra. Novos números foram gerados até completar o número previamente estabelecido $(n=6)$ de escolas da amostra. Convém registrar que as seis unidades de ensino sorteadas para integrar a amostra localizavam-se em áreas rurais do município. Tal situação possivelmente decorre do fato da população residir majoritariamente na zona rural de Piedade, tendência que também é observada no tocante a localização da maioria das unidades escolares integrantes da rede pública de ensino que se situa nesse estrato geográfico.

Cabe esclarecer que o número de seis unidades de ensino foi estabelecido, levando-se em consideração os recursos humanos, tempo para execução da pesquisa, características e número total das unidades que integram a rede pública de ensino. Nesse caso, o número de escolas pode ser considerado de conveniência. No entanto, os processos de seleção das unidades, bem como dos alunos que integraram a amostra, respeitaram critérios estatísticos, conforme descrito.

Dessa forma, participaram do estudo 150 alunos com idade entre 7 e 14 anos, sendo 45 alunos com idade inferior a 10 anos e 105 com idade entre 10 e 14 anos, de ambos os gêneros, matriculados nas escolas estaduais de ensino fundamental e médio, residentes, em sua maioria, em áreas rurais do município.

O sorteio dos alunos foi realizado em sala de aula, com auxílio da lista de chamada dos professores. Os alunos foram devidamente esclarecidos sobre o objetivo e procedimentos da pesquisa. Sortearam-se seis alunos de cada uma das classes mantidas nas unidades de ensino, exceto as classes do período noturno. Os termos de consentimento livre e esclarecido (TCLE) foram encaminhados aos pais ou responsável por intermédio dos alunos sorteados. É importante esclarecer que foram sorteados, inicialmente, seis alunos em cada uma das classes mantidas nos períodos matutino e vespertino por não se dispor, com antecedência, do número de alunos cujos pais ou responsável concordariam com a participação do filho na pesquisa. No entanto, já estava previsto, segundo conveniência dos pesquisadores, que dos seis alunos sorteados, apenas três participariam da pesquisa. No caso da devolução do TCLE, pelos seis escolares, novo sorteio foi realizado visando obter os nomes dos três alunos que permaneceriam na amostra final. Cabe destacar que, praticamente na totalidade das salas de aulas, foi observada aquiescência para que as crianças participassem da pesquisa de $100 \%$ dos pais ou responsável. A pesquisa foi aprovada pelo Comitê de Ética da Faculdade de Odontologia de Piracicaba - UNICAMP (protocolo 099/2004).

Os dados antropométricos de peso e altura foram coletados durante o mês de maio de 2005, com a participação da autora e dois nutricionistas, previamente treinados.

O peso foi obtido utilizando-se balança digital, marca CAMRY (ED-309), com capacidade para 120 quilogramas e sensibilidade de 500 gramas, devidamente aferida. Os integrantes da amostra foram posicionados com os pés centralizados, 
descalços e com o mínimo de roupa possível.

A altura foi obtida com os alunos em posição ereta, pés descalços, unidos e em paralelo, nádegas, ombros e a parte posterior da cabeça posicionados adequadamente no estadiômetro e os braços soltos ao longo do corpo. Foi registrada a recomendação para que, durante a aferição, o indivíduo se mantivesse na posição ereta, sem se encolher ou esticar, olhando para frente. Utilizou-se estadiômetro portátil, marca SECA (206), afixado em suporte de madeira apropriado, sendo este firmemente apoiado à parede, assegurando-se que a criança encontravase em posição correta para a leitura e registro da altura observada.

As medidas foram obtidas em duplicata por três pesquisadores e, para os cálculos dos índices antropométricos, foram utilizadas as médias destas medidas.

\section{Avaliação do Estado Nutricional}

Por meio da utilização do software EPI-INFO ${ }^{[13]}$, foi construído um banco de dados que viabilizou os cálculos e as análises, envolvendo os indicadores escore $Z$ de altura para idade $(Z A I)$, de peso para idade (ZPI) e Índice de Massa Corporal (IMC) ${ }^{[14]}$.

Os escolares que apresentaram ZAI $<-2$ foram identificados com altura inferior à esperada para idade e gênero. As crianças classificadas no intervalo de $-2 \leq Z \mathrm{AI}<-1$ foram identificadas em situação de risco, com discreto comprometimento da altura e quando o escore $Z$ for igual ou superior $a-1$, considera-se indicativo de eutrofia ${ }^{[14]}$.

Quanto ao escore $Z$ de peso para idade (ZPI), os escolares que apresentaram $Z \mathrm{PI}<-2$ foram identificados em situação de baixo peso para a idade. Para a indicação de eutrofia, considerou-se o nível crítico de $Z \mathrm{PI} \geq-1^{[14]}$.

Visando caracterizar a situação nutricional atual dos escolares, que possibilita a identificação da prevalência de obesidade, analisou-se a distribuição dos percentis do Índice de Massa Corporal (IMC). Para tanto, adotou-se, conforme preconizado pela World Health Organization - WHO ${ }^{[15]}$, os seguintes intervalos: $\mathrm{IMC} \leq 5^{\circ} \mathrm{P}$ (indicativo de baixo peso), 5 $\mathrm{P}<\mathrm{IMC}<95^{\circ} \mathrm{P}$ (indicativo de eutrofia) e IMC $\geq 95^{\circ} \mathrm{P}$ (indicativo de obesidade). O padrão de referência adotado é preconizado pelo Center for Disease Control and Prevention, do National Center for Health Statistics - NCHS ${ }^{[14]}$.

\section{Resultados}

A Tabela 1 apresenta a distribuição dos escolares integrantes da amostra, de acordo com o gênero e estrato de idade.

Tabela 1. Distribuição dos escolares da rede pública de ensino, de acordo com os estratos de idade e gênero. Piedade, São Paulo, 2005.

\begin{tabular}{|c|c|c|c|c|c|c|}
\hline \multirow{3}{*}{$\begin{array}{c}\text { Estratos de } \\
\text { idade } \\
\text { (anos) }\end{array}$} & \multicolumn{4}{|c|}{ Gênero } & \multirow{2}{*}{\multicolumn{2}{|c|}{ Total }} \\
\hline & \multicolumn{2}{|c|}{ Feminino } & \multicolumn{2}{|c|}{ Masculino } & & \\
\hline & $n$ & $\%$ & $n$ & $\%$ & $n$ & $\%$ \\
\hline $7-9$ & 27 & 29,0 & 18 & 31,6 & 45 & {$[30,0]$} \\
\hline $10-12$ & 43 & 46,3 & 23 & 40,3 & 66 & {$[44,0]$} \\
\hline $13-14$ & 23 & 24,7 & 16 & 28,1 & 39 & {$[26,0]$} \\
\hline Total & 93 & {$[62,0]$} & 57 & {$[38,0]$} & 150 & {$[100,0]$} \\
\hline
\end{tabular}

Obs.: Os números entre colchetes são os percentuais em relação ao total de escolares $(n=150)$ da amostra. 
$\mathrm{Na}$ seqüência, são mostrados os resultados relativos à situação nutricional, com base nos indicadores ZAI e ZPI (Tabela 2).

Tabela 2. Distribuição dos escolares de acordo com os indicadores antropométricos do estado nutricional. Piedade, São Paulo, 2005.

\begin{tabular}{|c|c|c|}
\hline \multirow{2}{*}{ Estado Nutricional } & \multicolumn{2}{|c|}{ Observações } \\
\hline & $n$ & $\%$ \\
\hline$Z \mathrm{AI}<-2$ & 6 & 4,0 \\
\hline$-2 \leq Z \mathrm{AI}<-1$ & 29 & 19,3 \\
\hline$-1 \leq Z \mathrm{AI}<2$ & 114 & 76,0 \\
\hline$Z A I \geq 2$ & 1 & 0,7 \\
\hline Total & 150 & 100,0 \\
\hline$Z \mathrm{PI}<-2$ & 3 & 2,0 \\
\hline$-2 \leq Z \mathrm{PI}<-1$ & 31 & 20,7 \\
\hline$-1 \leq Z \mathrm{PI}<2$ & 113 & 75,3 \\
\hline$Z \mathrm{PI} \geq 2$ & 3 & 2,0 \\
\hline Total & 150 & 100,0 \\
\hline
\end{tabular}

Observando-se o total de escolares, nota-se que $4 \%$ foram classificados com altura inferior para a idade $(Z A I<-2)$. A proporção identificada é praticamente o dobro da verificada em populações adotadas como referência.

Cabe ainda salientar que a proporção de escolares, classificada no intervalo $-2 \leq \mathrm{ZAI}<-1$, mostra-se superior a $13,6 \%$ (proporção esperada). Destaca-se, diante desta situação, a importância de monitorar o crescimento, com vistas à prevenção de situações que possam contribuir para o agravamento do estado nutricional dos escolares.

Quando se analisa a proporção de escolares com escore $Z$ de peso para idade ( $Z \mathrm{PI}$ ) inferior a 2 (situação indicativa de baixo peso), verifica-se que apenas $2 \%$ dos escolares foram identificados nesta condição. Trata-se de proporção praticamente idêntica à esperada. Assim, pode-se reconhecer a existência de situação satisfatória entre os alunos das escolas públicas de Piedade no tocante ao escore de peso para a idade. Quanto à eutrofia, discriminada por ZPI $\geq-1$, foram identificados $77,3 \%$ dos escolares nesta condição. Vale lembrar que é esperada a proporção de $84,1 \%$ de indivíduos (grupos considerados de referência) nesta situação.

Na Tabela 3, foram reunidos os resultados referentes à situação nutricional, com base no Índice de Massa Corporal (IMC).

Tabela 3. Distribuição dos escolares de acordo com o Índice de Massa Corporal (IMC). Piedade, São Paulo, 2005.

\begin{tabular}{|c|c|c|}
\hline \multirow{2}{*}{ In tervalo } & \multicolumn{2}{|c|}{ O b s ervaçõ es } \\
\hline & $n$ & $\%$ \\
\hline$\leq 5$ o $\mathrm{P}$ & 16 & 10,7 \\
\hline $5 \circ \mathrm{P}<\mathrm{IMC}<95^{\circ} \mathrm{P}$ & 124 & 82,7 \\
\hline$\geq 95 \circ \mathrm{P}$ & 10 & 6,6 \\
\hline Total & 150 & 100,0 \\
\hline
\end{tabular}

Em relação ao IMC, na situação indicativa de baixo peso (valor $\leq 5^{\circ} \mathrm{P}$ ), constatou-se $10,7 \%$ de escolares. Note-se que a proporção identificada é praticamente o dobro da esperada $(5 \%)$.

Merece atenção a proporção de escolares classificados com baixo peso, tendo por base o indicador ZPI <-2, que revelou ser inferior à proporção identificada pelo IMC. Contudo, o indicador ZPI, como mencionado anteriormente, não considera a altura observada, o que poderia justificar os resultados discordantes em relação ao baixo peso.

A análise da prevalência de escolares diagnosticados com IMC $\geq 95_{-}^{\circ} \mathrm{P}$, situação indicativa de obesidade, revela proporção de $6,6 \%$, valor ligeiramente superior ao esperado (5\%).

\section{Discussão}

Os resultados relativos aos alunos de Piedade, apresentados neste artigo, que tiveram por base dados de amostra probabilística, podem contribuir para ampliar, de forma geral, o acervo de informações sobre a situação nutricional da população escolar do país e, adicionalmente, descrever a situação de grupos de alunos que sejam oriundos de municípios que reúnam características similares àquelas identificadas em Piedade, ou seja, 
população predominantemente moradora do meio rural e expressivo número de unidades de ensino e alunos também residentes nesse estrato geográfico.

Os resultados de pesquisas sobre o estado nutricional do público infantil e jovem revelam contrastes importantes, especialmente quando se considera a população das diversas regiões do país e o local de moradia (rural ou urbano).

Ilustram parcialmente tais contrastes, os dados coletados durante a realização da Pesquisa de Orçamentos Familiares (POF) 2002-2003 e publicados pelo IBGE, tendo como foco o estado nutricional de crianças e adolescentes brasileiros. Em relação aos adolescentes (faixa etária compreendida entre 10 e 19 anos), os resultados revelaram declínio intenso e contínuo na freqüência de déficit de altura para idade ao longo dos três inquéritos realizados (ENDEF, PNSN e POF-2002-2003). Por outro lado, o excesso de peso apresentou situação inversa, sendo que na pesquisa realizada pelo ENDEF 5,7\% dos adolescentes (ambos os gêneros) revelavam excesso de peso e, no início do século XXI, 16,7\% dos adolescentes apresentaram a mesma situação ${ }^{[16]}$.

Diferenças regionais também foram constatadas por meio dos dados da POF 2002-2003. Entre os adolescentes, a prevalência de excesso de peso foi maior nas regiões Sul $(22,6 \%)$, Sudeste $(21,5 \%)$ e Centro-Oeste $(19,3 \%)$ do que nas regiões Norte $(15,3 \%)$ e Nordeste $(11,8 \%)$. A prevalência de excesso de peso também foi maior para as meninas das regiões Sul (17\%), Sudeste $(18 \%)$ e Centro-Oeste $(15,6 \%)$, quando comparada com as regiões Norte $(13,9 \%)$ e Nordeste $(11,6 \%)$. Ao observar os dados de acordo com o estrato geográfico, para as cinco regiões, tanto para meninos quanto para meninas, o excesso de peso foi mais prevalente no meio urbano do que no meio rural. A menor proporção de excesso de peso foi constatada entre os meninos moradores do Nordeste rural $(6,8 \%)$ e a maior no Sul urbano $(23,6 \%)^{[16]}$.

Quando se considera a realidade da população residente no meio rural do país, cabe registrar que Veiga \& Burlandy ${ }^{[17]}$ analisaram dados obtidos junto à amostra de indivíduos residentes em áreas rurais (assentamento no Estado do Rio de Janeiro) e observaram 4,0\% de déficit estatural entre crianças e adolescentes com idade entre zero e 17,9 anos.

Ainda, segundo os referidos autores, é possível observar que a reduzida prevalência de déficit nutricional é contrastante com os resultados obtidos por meio de pesquisas nacionais. Os autores relatam que esse perfil mais favorável das crianças, residentes no assentamento alvo da pesquisa, possa estar relacionado ao fato de que cerca da metade das famílias com crianças e adolescentes fez referência a algum tipo de atendimento médico. É importante mencionar também que, além disso, a proximidade da residência do centro urbano amplia as chances de atendimentos médicos, incluindo também o acesso a campanhas de vacinação. A alteração nutricional de maior relevância, identificada pelos referidos autores, foi o sobrepeso $(7,9 \%) \mathrm{com}$ maior prevalência entre os adolescentes.

Balaban \& Silva ${ }^{[18]}$ identificaram situação distinta dos dados obtidos na presente pesquisa. Os referidos autores registraram prevalência maior de sobrepeso e obesidade entre os integrantes da amostra (escolares com idade entre 7 e 15 anos).

Koga ${ }^{[19]}$, tendo por base amostra de escolares com idade entre 7 e 10 anos, matriculados em unidades de ensino da cidade de São Paulo, identificou reduzida prevalência de baixo peso $(4,5 \%)$ e proporções de escolares com sobrepeso (identificado pelo intervalo $85^{\circ} \mathrm{P} \leq \mathrm{IMC}<95^{\circ} \mathrm{P}$ ) de $15,3 \%$ e obesidade (IMC $\geq 95^{\circ}$ P) de $11 \%$ (proporção que é mais que o dobro da esperada $5 \%$ ).

Pesquisa baseada em amostra $(n=401)$ de alunos ( $5^{\underline{a}}$ a $8^{\underline{a}}$ séries), matriculados em escolas das redes municipal, estadual e particular de Ribeirão Preto (SP), viabilizou a identificação de proporções de $12,6 \%$ e $8,5 \%$ de escolares classificados com sobrepeso e obesidade, respectivamente ${ }^{[20]}$.

Pesquisa implementada em Porto Calvo ${ }^{[21]}$, Estado de Alagoas (área invadida pelo "Movimento dos Sem Terra"), envolvendo amostra de crianças com idade inferior a 10 anos, mostrou prevalência de déficit de altura $(Z A I \leq-2)$ que alcançou 39,8\%. Ferreira et al. ${ }^{[21]}$ destacam que a elevada freqüência 
de indivíduos de baixa estatura indica que, historicamente, a população não tem acesso aos meios necessários para seu adequado crescimento e desenvolvimento. Ainda de acordo com Ferreira et al. ${ }^{[21]}$, os resultados observados superam valores divulgados em 1993, pelo Unicef, tendo como referência a zona rural do Estado de Alagoas. Caso fosse adotada como referência a pesquisa do Unicef, a expectativa era de identificar $22,7 \%$ de indivíduos com déficit de altura.

No Estado da Paraíba, Brasil, foi implementado levantamento de natureza censitária, da estatura de 50.144 escolares, com idade entre 6 e 9 anos, matriculados na primeira série do ensino fundamental. Integrou a pesquisa a totalidade dos municípios do Estado. Os autores adotaram como parâmetro para a avaliação do déficit estatural valores classificados abaixo de -2 escores $Z$. Em todo o Estado da Paraíba, foram identificados 14,5\% de escolares com déficit estatural. De acordo com os autores, $17,8 \%$ dos alunos matriculados nas escolas da área rural apresentavam déficit estatural, enquanto no meio urbano a prevalência deste distúrbio alcançou $11,8 \%{ }^{[22]}$.

Castro et al. ${ }^{[23]}$ avaliaram a situação nutricional de crianças com idade entre zero e 60 meses, residentes em um assentamento rural na região do Vale do Rio Doce, Estado de Minas Gerais. Os resultados revelaram que $7,6 \%$ das crianças apresentavam déficits, quando foram analisados os índices peso/idade e estatura/idade (índice menor que a média nacional de 10,5\% para crianças menores de 5 anos), com a adoção do critério escore- $Z$. A maior proporção de desnutridos foi encontrada entre as crianças com idade entre 1 e dois anos. Tal tendência já havia sido identificada nas análises envolvendo os dados da Pesquisa Nacional sobre Demografia e Saúde - PNDS [23].

Os resultados obtidos na presente pesquisa podem ser, em parte, comparados com os dados obtidos por meio da pesquisa realizada no ano de 2000 por Maestro ${ }^{[24]}$, também no município de Piedade. $\mathrm{O}$ autor teve como objetivo conhecer o estado nutricional, o consumo alimentar e o estilo de vida de amostra de escolares, matriculados na rede pública de ensino, com idade entre 6 e 18 anos.
Em relação ao estado nutricional, foi constatado que $3 \%$ dos escolares apresentavam comprometimento estatural $(Z A I<-2)$.

Transcorridos cinco anos entre a realização da pesquisa de Maestro ${ }^{[24]}$ e o presente estudo, merece atenção a discreta redução na prevalência do déficit de altura e na situação de risco nutricional entre os escolares de Piedade. Note-se que, em 2000, foram identificados $25 \%$ (proporção superior à esperada - 13,6\%) de alunos da amostra com o referido distúrbio. Na presente pesquisa, foram classificados $19,3 \%$ de escolares no intervalo que possibilita a identificação da prevalência de déficit da altura.

A proporção de escolares identificados como eutróficos $(Z A I \geq-1)$ atingiu $76,7 \%$ (valor inferior ao esperado - 84,1\%). Maestro ${ }^{[24]}$ encontrou $69,7 \%$ de indivíduos nesta situação.

Em relação às análises envolvendo o IMC, Maestro ${ }^{[24]}$ identificou $10 \%$ de escolares com IMC $\geq 85^{\circ} \mathrm{P}$ (situação que engloba indivíduos com sobrepeso e obesidade), enquanto que os dados da presente pesquisa revelam $6,6 \%$ de escolares com $\mathrm{IMC} \geq 95^{\circ} \mathrm{P}$, indicativo apenas de obesidade. Portanto, pode-se inferir que a proporção de escolares que é indicativa do grupo com excesso de peso esteja subestimada por não ter sido considerada a prevalência referente ao sobrepeso.

Maestro ${ }^{[24]}$ encontrou $22,1 \%$ de escolares classificados abaixo do $15^{\circ} \mathrm{P}$ (nível crítico para discriminação de baixo peso), o que revela a existência de 7,1 pontos percentuais acima do esperado $(15 \%)$. Na atual pesquisa, utilizou-se o limite de IMC $\leq$ 5 $\mathrm{P}$ como indicativo de baixo peso, observando-se proporção de $10,7 \%$ de indivíduos nesse intervalo. Tal proporção é o dobro da esperada $(5 \%)$.

Pode-se observar, nesta pesquisa, que a proporção de escolares com baixo peso supera a prevalência de escolares com IMC $\geq 95^{\circ}$ P. É possível notar que os resultados do município de Piedade diferem de resultados freqüentemente obtidos junto a escolares matriculados na rede pública de ensino de expressiva parcela de municípios paulistas. No entanto, cabe registrar que a maioria das pesquisas 
privilegia, de maneira tradicional, amostra de alunos exclusivamente moradores do meio urbano. Contudo, mesmo revelando reduzida prevalência, o sobrepeso e a obesidade entre adolescentes não devem ser subestimados, visto que há um risco aumentado de persistirem na vida adulta ${ }^{[25]}$.

De acordo com os resultados obtidos pela POF 2002-2003 ${ }^{[16]}$, os quais discriminam os escolares de acordo com a situação do domićlíio, nota-se que a proporção de escolares da região Sudeste, residentes em áreas rurais, classificados em baixo peso, é de 7,1\% para o grupo dos meninos e 10,1\% para as meninas. Quanto ao excesso de peso, 17,4\% dos meninos e $16,4 \%$ das meninas, residentes também em áreas rurais da região Sudeste, foram classificados em tal condição. Vale registrar que nas áreas urbanas da região Sudeste, $22 \%$ dos meninos e $18,2 \%$ das meninas foram identificados em excesso de peso.

Destaca-se que as proporções de escolares com déficit de altura, bem como daqueles identificados em situação de discreto comprometimento da altura, demandam monitoramento constante. Torna-se importante evitar situações que favoreçam o agravo do estado nutricional e estimular a adoção de medidas para intervir e preferencialmente prevenir tal condição.

Tendo em vista que a adolescência é definida como um fenômeno biopsicossocial, o impacto da transição nutricional, bem como do estilo de vida mais sedentário observado entre a população residente nos grandes centros urbanos, revela-se menos marcante nas áreas rurais. Convém registrar que o número de pesquisas, especialmente envolvendo os hábitos alimentares e o potencial efeito para a saúde, em populações rurais no Brasil, ainda é considerado escasso.

Dessa forma, análises envolvendo também populações residentes em áreas rurais tornam-se importantes por representarem, às vezes, fontes de informações inéditas, oferecendo subsídios para o planejamento de ações de saúde bem focalizadas e eficazes.

Propõe-se a intensificação de estudos sobre as condições de saúde da população infantil e também adulta, residente nesses locais, com a inclusão de objetivos visando ao diagnóstico do consumo de alimentos, estado nutricional e informações referentes ao estilo de vida. A iniciativa possibilitaria a elucidação de fatores que possam influenciar o desenvolvimento de desequilíbrios nutricionais, a curto ou longo prazo, o que inclui as doenças crônico-degenerativas associadas à alimentação.

\section{Conclusão}

Os resultados dos escolares do município mostraram que predomina o comprometimento de altura entre as crianças e adolescentes matriculados nas escolas públicas. A proporção identificada do distúrbio é praticamente o dobro da porcentagem esperada (cerca de 2,0\%). Este resultado contribui para confirmar que os déficits de altura ainda acompanham as crianças e jovens brasileiros, mesmo aqueles residentes nas regiões geográficas mais ricas do País. Prevalência de baixo peso (o dobro da proporção esperada) entre os alunos também foi identificada. No que diz respeito à obesidade, a proporção observada é praticamente a metade daquela verificada em grupos de referência. Os resultados relativos aos déficits de peso, assim como a situação oposta - a obesidade, parecem guardar relativa distância do processo que vem se intensificando e predominando na maioria das regiões brasileiras, com destaque para as localidades onde a população ocupa, na grande maioria, as áreas urbanas e ou metropolitanas.

\section{Considerações Finais}

Por meio da presente pesquisa, foi possível caracterizar o estado nutricional de amostra de escolares da rede pública de ensino do município de Piedade (SP).

É importante enfatizar que o ambiente escolar pode constituir um importante espaço para o desenvolvimento de estratégias de intervenção. Obviamente que outras medidas, em curtíssimo prazo, entre elas a geração de emprego e renda, também devem ser contempladas, tendo em vista as substanciais diferenças observadas quando, por exemplo, são levados em consideração os níveis de escolaridade e rendimentos familiares entre 
moradores do meio urbano e aqueles residentes no meio rural.

\section{Referências Bibliográficas}

[1] Fernandes BS. Nova abordagem para o grave problema da desnutrição infantil. Est Avanç 2003;17(48):77-92.

[2] Monteiro CA. A dimensão da pobreza, da desnutrição e da fome no Brasil. Est Avanç 2003;17(48):7-20.

[3] Batista Filho M, Rissin A. A transição nutricional no Brasil: tendências regionais e temporais. Cad Saúde Pública 2003;19(1):181-191.

[4] Sawaya AL, Solymos GMB, Florêncio TMMT, Martins PA. Os dois Brasis: quem são, onde estão e como vivem os pobres brasileiros. Est Avanç 2003;17(48):21-44.

[5] Laurentino GEC, Arruda IKG, Arruda BKG. Nanismo nutricional em escolares no Brasil. Rev Bras Saúde Matern Infant 2003;3(4):377-385.

[6] Oliveira AMA, Cerqueira EMM, Souza JS, Oliveira AC. Sobrepeso e obesidade infantil: influência de fatores biológicos e ambientais em Feira de Santana, BA. Arq Bras Endocrinol Metab 2003;47(2):144-150.

[7] Instituto Brasileiro de Geografia e Estatística. Análise da disponibilidade domiciliar de alimentos e do estado nutricional no Brasil. [citado 2006 maio 20]. Disponível em: http://www.ibge.gov.br/ home/estatistica/populacao/condicaodevida/pof/ 2002analise/default.shtm

[8] Repetto G, Rizzolli J, Bonatto J. Prevalência, riscos e soluções na obesidade e sobrepeso: here, there, and everywhere. Arq Bras Endocrinol Metab 2003;47(6):633-635.

[9] Dietz WH. Overweight in childhood and adolescence. N Engl J Med 2004;350(9):855-857.

[10] Castro MPS. Hábitos alimentares de estudantes brasileiros das zonas urbana e rural: um estudo de
Garça - São Paulo [dissertação]. São Paulo: Universidade de São Paulo; 2001.

[11] Instituto Brasileiro de Geografia e Estatística. Censo 2000. [citado 2005 jul 09]. Disponível em: http://www.ibge.gov.br

[12] Instituto de Pesquisas Educacionais Anísio Teixeira - INEP. Resultados finais do censo escolar de 2004. [citado 2005 jul 09]. Disponível em: http:/ /www.inep.gov.br

[13] Dean AG, Dean JA, Burton AH, Dicker RC. Epi Info 2000: a database and statistics program for public health professionals for use on Windows 95, 98, NT and 2000 computers [CD-ROM]. Atlanta: Centers for Disease Control and Prevention; 2000.

[14] National Center for Health Statistics. Center for Disease Control and Prevention. Growth Charts. [cited 2005 Jul 09]. Disponível em: http:// www.cdc.gov/growthcharts

[15] World Health Organization. Physical status: the use and interpretation of anthropometry. Technical Report Series, 854. Geneva; 1995.

[16] Instituto Brasileiro de Geografia e Estatística. Pesquisa de Orçamentos Familiares: antropometria e análise do estado nutricional de crianças e adolescentes no Brasil. [citado 2007 fev 12]. Disponível em: http://www.ibge.gov.br/home/ estatistica/populacao/condicaodevida/pof/ 2003medidas/default.shtm

[17] Veiga GV, Burlandy L. Indicadores sócioeconômicos, demográficos e estado nutricional de crianças e adolescentes residentes em um assentamento rural do Rio de Janeiro. Cad Saúde Pública 2001;17(6):1465-1472.

[18] Balaban G, Silva GAP. Prevalência de sobrepeso e obesidade em crianças e adolescentes de uma escola da rede privada de Recife. Jornal de Pediatria 2001;77(2):96-101.

[19] Koga CR. Estado nutricional de escolares de 7 a 10 anos de idade: diagnóstico e comparação de 
métodos [dissertação]. São Paulo: Universidade de São Paulo; 2005.

[20] Zancul MS. Consumo alimentar de alunos nas escolas de ensino fundamental em Ribeirão Preto (SP) [dissertação]. Ribeirão Preto: Faculdade de Medicina de Ribeirão Preto; 2004.

[21] Ferreira HS, Albuquerque MFM, Ataíde TR, Morais MGC, Mendes MCA, Siqueira TCA, Silva GJ. Estado nutricional de crianças menores de dez anos residentes em invasão do "Movimento dos Sem Terra", Porto Calvo, Alagoas. Cad Saúde Pública 1997;13(1):137-139.

[22] Carvalho AT, Costa MJC, Ferreira LOC, Batista Filho M. Cartografia do retardo estatural em escolares do Estado da Paraíba, Brasil. Rev Saúde Pública 2000;34(1):3-8.

[23] Castro TG, Campos FM, Priore SE, Coelho FMG, Campos MTFS, Franceschini SCC, Rangel AA. Saúde e nutrição de crianças de 0 a 60 meses de um assentamento de reforma agrária, Vale do Rio
Doce, MG, Brasil. Rev Nutr 2004;17(2):167-176.

[24] Maestro V. Padrão alimentar e estado nutricional: caracterização de escolares de município paulista [dissertação]. Piracicaba: Escola Superior de Agricultura Luiz de Queiroz; 2002.

[25] Serdula MK, Ivery D, Coates RJ, Freedman DS, Williarnson DF, Byers T. Do obese children become obese adults? - A review of the literature. Prev Med 1993;22:167-177.

\section{Autores}

Giovana Eliza Pegolo - Doutoranda do Departamento de Alimentos e Nutrição / Ciências Nutricionais, Faculdade de Ciências Farmacêuticas - UNESP, Araraquara-SP.

Correspondência: gpegolo@hotmail.com

Marina Vieira da Silva - Professora Doutora do Departamento de Agroindústria, Alimentos e Nutrição Escola Superior de Agricultura "Luiz de Queiroz", ESALQ-USP, Piracicaba-SP.

Recebido em: 25/09/2007

Aceito em: 12/05/2008 